\title{
Euglycemic ketoacidosis complicating empagliflozin therapy in a Bangladeshi type 2 diabetic lady
}

\author{
Laboni Ra , Afsana Fb, Hossain KN c,Rouf RBc, Amin MFd, Ashrafuzzaman SMe, \\ Pathan MFe
}

\begin{abstract}
Diabetic ketoacidosis (DKA) is an acute complication of diabetes mellitus, which may occurin both type 1 and type 2 diabetic patients. It is characterized by hyperglycaemia [blood glucose levels $>250 \mathrm{mg} / \mathrm{dL}(13.8 \mathrm{mmol} /$ L)], metabolic acidosis ( $\mathrm{pH}<7.3$ or serum bicarbonate $<15 \mathrm{mmol} / \mathrm{L}$ ) with an increased anion gap and the presence of ketone bodies in blood or urine. A subgroup of patients may present without signs of hyperglycemia, rather present with euglycemia, posing a diagnostic challenge due to the absence of the cardinal findings of the pathophysiology in DKA. Here, we report a case of euglycemic ketoacidosis complicating a 50-year-old Bangladeshi diabetic lady receiving empagliflozin treatment.
\end{abstract}

Key words: euglycemic ketoacidosis, SGLT2 inhibitors induced ketoacidosis, empagliflozin.

(BIRDEM Med J 2021; 11(3): 243-245)

\section{INTRODUCTION}

Diabetic ketoacidosis (DKA) is a serious and potentially life threatening acute complication of diabetes mellitus (DM), characterized by ketoacidosis and hyperglycemia. Patients with euglycemic ketoacidosis, however, present with a plasma glucose of $<200 \mathrm{mg} / \mathrm{dL}^{1}$ and is therefore, a challenge to identify in the emergency department. Absence of marked hyperglycemia often leads to delayed

\section{Author Information}

a. Rezwana Laboni, MD (Thesis Part) Student, Department of Endocrinology, BIRDEM General Hospital, Dhaka, Bangladesh.

b. Faria Afsana, Assistant Professor, Department of Endocrinology, BIRDEM General Hospital, Dhaka, Bangladesh.

c. Kazi Nazmul Hossain, Rushda Sharmin Binte Rouf, Registrar, Department of Endocrinology, BIRDEM General Hospital, Dhaka, Bangladesh.

d. Mohammod Feroz Amin, Assoaciate Professor, Department of Endocrinology, BIRDEM General Hospital, Dhaka, Bangladesh.

e. S. M. Ashrafuzzaman, Md. Faruque Pathan, Professor, Department of Endocrinology, BIRDEM General Hospital, Dhaka, Bangladesh.

Address of correspondence: Faria Afsana, Assistant Professor, Department of Endocrinology, BIRDEM General Hospital, Dhaka, Bangladesh. Email: fariaafsana@yahoo.com Received: July 3, 2021

Revision received: July 7, 2021

Accepted: July 31, 2021 diagnosis of euglycaemic ketoacidosis. Besides being reported in type 1 diabetic patients and pregnant women, euglycemic ketoacidosis has also recently been reported to be associated with treatment of sodiumglucose cotransporter 2 (SGLT2) inhibitors, one of the newest classes of antidiabetic medications that achieve glycemic control by inhibiting renal glucose reabsorption and promoting glycosuria. Due to glycosuria, plasma glucose level falls, glucose dependent insulin release decreases and also increases glucagon secretion. Insulin deficiency causes lipolysis, free fatty acid promotes ketogenesis in liver, resulting in euglycemic ketoacidosis. Here, we report case history of a type 2 diabetic patient, who presented with euglycemic ketoacidosis, possibly, secondary to the administration of empagliflozin.

\section{CASE REPORT}

A 50-year-old Bangladeshi lady, known case of type 2 diabetes mellitus (for 5 years), presented with fever, dry cough, anorexia and burning sensation during micturition for 7 days. She also complained of generalized weakness and her blood glucose was grossly uncontrolled. Her food intake was inadequate for the preceding 7 days. She was also diagnosed as having adjustment disorder 1 year back, after the death of her 
husband. She was getting gliclazide $(120 \mathrm{mg})$ in two divided doses and combination of linagliptin and metformin $(2.5 / 500 \mathrm{mg}$ ) once daily. Empagliflozin $(25 \mathrm{mg})$ once daily was added to her ongoing medications 4 days prior to admission to our center with features of urinary tract infection (UTI). She also received ceftriaxone and linezolid prior to her admission.

Vitals at presentation in our facility revealed a temperature of $98.6^{\circ} \mathrm{F}$, pulse $112 / \mathrm{min}$, blood pressure $90 / 60 \mathrm{~mm}$ of $\mathrm{Hg}$, respiratory rate 18 breath/min, $\mathrm{SpO} 2$ $100 \%$ in room air. She was ill looking, was dehydrated with dry oral mucosa and poor skin turgor. Arterial blood gas analysis revealed severe metabolic acidosis with $p \mathrm{H} 6.965, \mathrm{HCO}_{3}$ was $4.2 \mathrm{mmol} / \mathrm{L}$, anion gap $12.2 \mathrm{mmol} / \mathrm{L}$, serum creatinine was $1.2 \mathrm{mg} / \mathrm{dl}$ and blood urea was 41 $\mathrm{mg} / \mathrm{dl}$. Her blood glucose was $10.1 \mathrm{mmol} / \mathrm{L}$ (181.8 mg/ $\mathrm{dL}$ ), urine for acetone was ++ , urine routine examination revealed pus cell 10-15/high power field and urine culture grew Candida albicans. There was neutrophil leukocytosis (total white cells $15640 / \mathrm{mm}^{3}$, neutrophils 87.6\%), high C-reactive protein (CRP) (142 mg/l), D-dimer was $2.62 \mathrm{micro} \mathrm{gm} / \mathrm{ml}$, lactate dehydrogenase (LDH) was high (1105 U/L), serum ferritin was $548 \mathrm{ng} / \mathrm{ml}$. Chest radiograph was normal. Reverse transcriptase polymerase chain reaction (RT-PCR) for severe acute respiratory syndrome coronavirus type 2 (SARS-CoV2) appeared negative.

Patient was treated for euglycaemic ketoacidosis through intravenous rehydration therapy and insulin infusion. All the oral anti-diabetic agents including empagliflozin was stopped after admission. Serial blood gas analyses showed resolution of ketoacidosis (Table I) and clearance of urine ketones. Patient was discharged from hospital after 7 days, with advice for permanent cessation of empagliflozin administration.

Table I Serial arterial blood gas analysis of the patient with euglycemic ketoacidosis

\begin{tabular}{lccc}
\hline Parameter & \multicolumn{3}{c}{ Date } \\
& $30 / 12 / 20$ & $31 / 12 / 20$ & $2 / 1 / 21$ \\
\hline$p \mathrm{H}$ & 6.965 & 7.224 & 7.4796 \\
$\mathrm{PCO}_{2}(\mathrm{~mm} \mathrm{Hg})$ & 16.4 & 19.9 & 35.3 \\
$\mathrm{HCO}_{3}(\mathrm{mmol} / \mathrm{L})$ & 4.2 & 8.2 & 26 \\
Base excess $(\mathrm{mmol} / \mathrm{L})$ & - & -17.4 & 2.6 \\
\hline
\end{tabular}

\section{DISCUSSION}

Empagliflozin is a selective SGLT2 inhibitor approved to treat DM, either singly or in combination with other antidiabetics. Experiences with empagliflozin-associated euglycemic ketoacidosis are not common. ${ }^{2}$ Being rarely observed in type 2 diabetes, euglycemic ketoacidosis can prove to be a diagnostic challenge for physicians unfamiliar with this class of antidiabetics, especially, since ketone studies and blood gas analysis are not available in many low resource settings. Seemingly normal blood glucose levels may also convey a false impression of the patient's clinical stability and delay treatment. DKA is classically defined as presence of the triad of hyperglycemia ( $>250 \mathrm{mg} / \mathrm{dL}$ ), ketosis and aniongap acidosis. ${ }^{3}$ Euglycemic ketoacidosis is DKA without marked hyperglycemia. Indeed, our patient had ketoacidosis, though the blood glucose level of 181.8 $\mathrm{mg} / \mathrm{dLwas}$ far below the usual means of that in the "traditional" DKA - the diagnosis would thus have been missed, if we had ruled out DKA based on the absence of marked hyperglycemia.

Euglycemic ketoacidosis in type 1 diabetic patients is primarily due to reduced carbohydrate availability with concomitant reduction in insulin dosage. Euglycemic ketoacidosis in type 2 patients on empagliflozin, however has a different pathophysiology-SGLT2 inhibition causes a sharp spike in the urinary excretion of glucose. Since insulin release is dependent on glucose, pancreatic release of insulin decreases correspondingly. Reduced inhibition by insulin, coupled with decreased SGLT2-mediated glucose transport into alfa-cells, then lead to significant increase in plasma glucagon levels. These shifts in hormones enable gluconeogenesis in the liver and augment endogenous glucose production. Paradoxically, insulin sensitivity is ultimately improved, limiting the rise in plasma glucose concentration. ${ }^{2}$ While these changes in glucose homeostasis are ongoing, insulin deficiency causes a concurrent increase in lipolysis from peripheral fat tissues, releasing free fatty acids. These fatty acids are subsequently converted into acetyl-CoA via betaoxidation by hepatic mitochondriaand acetyl-CoA molecules enters the ketogenic metabolic cycle to produce acetoacetic acid. Acetoacetic acid is then reduced to betahydroxybutyricacid; accumulation of these two acids results inan elevated anion gap metabolic acidosis. Acetoacetic acid is also 
decarboxylated to acetone, a ketone body which serves as an alternative energy source for patients in their state of reduced intracellular glucose availability secondary to insulin deficiency. ${ }^{1}$ All these metabolic processes thus ultimately manifest as euglycemic ketoacidosis, which was seen in our patient.

Decreased oral intake, as was seen in our patient, is a risk factor for SGLT2 inhibitor-related euglycemic ketoacidosis. Other possible precipitants include alcohol intake, surgery or perioperative preparations, insulin dose reduction or cessation, infections, hepatic or renal impairments, acute coronary events and pancreatitis. ${ }^{3-5}$ Other adverse effects of SGLT2 inhibitors are genitourinary tract infection, weakness and lethargy. ${ }^{6}$

Our patient developed euglycemic ketoacidosis 4 days after initiation of empagliflozin. Whereas in a case report, euglycemic ketoacidosis was reported 189 days after initiation of empagliflozin. ${ }^{7}$ In a retrospective analysis of randomized phase 2 and 3 empagliflozin trials $(>13,000$ type 2 diabetic patients), there were eight events consistent with DKA with no imbalance between patients treated with empagliflozin $10 \mathrm{mg}$ (two events), empagliflozin $25 \mathrm{mg}$ (one event) and placebo (five events). In the cardiovascular outcome trial EMPA-REG outcome with approximately 7000 patients, the frequency of reported blinded events of DKA was less than $0.1 \%{ }^{2}$

\section{Conclusion}

We report a case of life-threatening euglycemic ketoacidosis as a possible complication of empagliflozin treatment, a challenging and easily missed diagnosis. Such a presentation is very rare, nevertheless physicians are reminded to consider the diagnosis of euglycemic ketoacidosis in a patient whose drug regimen includes any SGLT2 inhibitor, especially if the patient presents with nausea, vomiting, abdominal pain, dyspnea, lethargy and dehydration. These patients should then be investigated with ketone studies and blood gas analyses regardless of blood glucose levels for prompt diagnosis and treatment. Patients started on SGLT2 inhibitors can also be counseled to perform urine dipstick tests to check for ketones, if they feel unwell and seek medical treatment immediately, if positive.

Authors' contribution: RL drafted manuscript, FA reviewed the manuscript. All the authors took part in clinical decision making, read and approved the final manuscript for publication.

Consent: Informed consent was taken from patient.

Conflicts of interest: Nothing to declare.

\section{REFERENCES}

1. Ogawa W, Sakaguchi K. Euglycemic diabetic ketoacidosis induced by SGLT2 inhibitors: possible mechanism and contributing factors. J Diabetes Invest 2016;7: 135-8.

2. Rosenstock J, Ferrannini E. Euglycemic diabetic ketoacidosis: a predictable, detectable, and preventable safety concern with SGLT2 inhibitors. Diabetes Care 2015;38:1638-42.

3. Peters AL, Buschur EO, BuseJB,Cohan P, Diner JC, Hirsch IB. Euglycemia diabetic ketoacidosis: apotential complication of treatment with sodium-glucose cotransporter inhibition. Diabetes Care 2015;38:168793.

4. Meyer EJ, Gabb G, Jesudason D. SGLT2 inhibitor-associated euglycemic diabetic ketoacidosis: a South Australian clinical case series and Australian spontaneous adverse event notifications. Diabetes Care 2018;41:e47-9.

5. FARXIGA (Dapagliflozin) [Package Insert]. Princeton, NJ: Bristol-MyersSquibb; 2014.

6. Harmandeep KG, Prajeet K, Sharma M, Ambrish M. Adverse effect profile and effectiveness of SGLT2 inhibitor- a prospective real world setting study. Indian JEndocrin Met 2019 Jan-Feb: 23 (1): 50-5.

7. Farjo PD, Kidd KM, Reece JL. A case of Euglycemic Diabetic Ketoacidosis Following long term Empagliflozin therapy. Diabetes Care 2016; 39: e165- e166. 